\title{
FMH-Tagung - Mengenausweitung aufgrund von DRG?
}

\author{
Beatrix Meyera , Barbara Rohner \\ a Leiterin Abteilung Tarife und Gesundheitsökonomie Spitalärzte \\ b Wissenschaftliche Mitarbeiterin Abteilung Tarife und Gesundheitsökonomie Spitalärzte
}

\author{
Immer wieder berichten Medien davon, in den Spitälern würden unnötige Behand- \\ lungen durchgeführt. Dabei werden oft Fallpauschalen als Ursache für eine Mengen- \\ ausweitung genannt. Was ist von solchen Aussagen zu halten? Vier Experten nah- \\ men dazu an der letzten FMH-Tagung Stellung.
}

An der vergangenen Tagung des Departements Tarife und Gesundheitsökonomie Spitalärzte der FMH präsentierten vier Experten ihre Einschätzung zum Thema Mengenentwicklung unter DRG und stellten diese dem interessierten Plenum zur Diskussion. Prof. Dr. med. Reinhard Busse von der Technischen Universität Berlin stützte sich dabei auf die Ergebnisse der wissenschaftlichen Begleitforschung zur Mengenentwicklung in Deutschland, bei welcher er als Co-Autor mitwirkte [1]. Zur Situation in der Schweiz berichteten Oliver Peters, Vizedirektor des Bundesamts für Gesundheit, sowie die beiden Fachärzte Dr. med. Stephan Heinz, Vorstandsmitglied der Schweizerischen Gesellschaft für Orthopädie und Traumatologie, und Dr. med. Urs Kaufmann, Präsident der Schweizerischen Gesellschaft für Kardiologie.

\section{Datenlage zeigt unterschiedliche Tendenzen}

Busse legte für die Schweiz und Deutschland dar, dass die Anzahl Spitalaufenthalte pro 100 Einwohner in den letzten Jahren stärker zugenommen hat als in anderen europäischen Ländern. Die Verweildauer verkürzte sich hingegen. In Deutschland nahmen die Fallzahlen insbe-

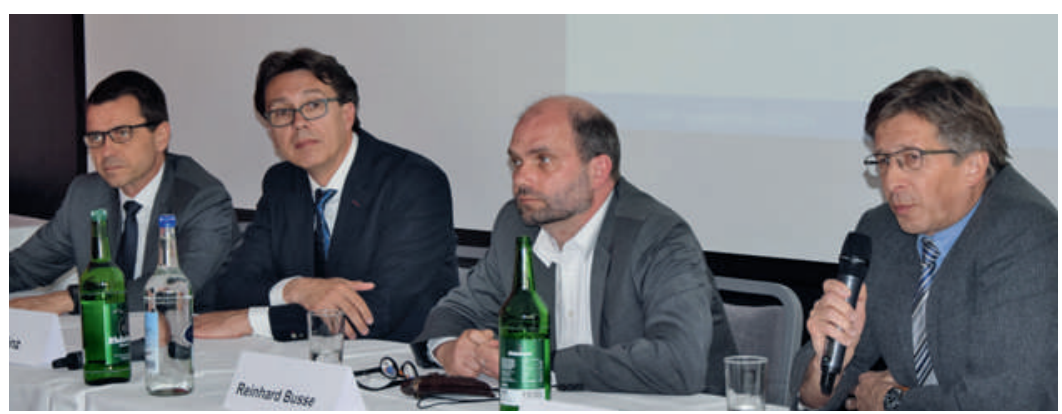

Die Referenten Peters, Heinz, Busse und Kaufmann bei der anschliessenden Diskussionsrunde. sondere von Krankheiten und Störungen am MuskelSkelett-System und Bindegewebe sowie diejenigen von Krankheiten und Störungen des Kreislaufsystems zu. Ein Mengenwachstum im stationären Bereich besteht in der Schweiz gemäss Peters insbesondere bei Notfallbehandlungen von über 70-jährigen Patientinnen und Patienten.

Laut Heinz ist im Kanton Thurgau in den letzten fünf Jahren gesamthaft keine Fallzahlsteigerung bei primären Implantationen von Hüft- und Knie-Totalprothesen festzustellen. In einer Privatklinik stieg nach deren Aufnahme auf die Spitalliste die Fallzahl zwar an, dafür sanken die Zahlen insgesamt in den beiden öffentlichen Spitälern. In der Kardiologie nahmen schweizweit die invasiven Fallzahlen gemäss Kaufmann in den letzten Jahren insgesamt erheblich zu. Auf der einen Seite haben die älteren Herzkatheter-Labors ihre Fallzahlen zwar nicht erhöht; auf der anderen Seite wurden in den letzten Jahren neue Labors eröffnet, was zusätzliche Fälle generierte. Kaufmann betont jedoch, dass es unklar sei, ob die Mengenausweitung aufgrund von DRG oder aus anderen Gründen erfolge, denn in der Schweiz fehlt eine entsprechende Begleitforschung.

\section{Vielschichtige Ursachen}

Aus Sicht von Peters ist die Mengenausweitung einerseits angebotsgetrieben, beispielsweise aufgrund von Überkapazitäten oder finanziellen Fehlanreizen. Andererseits ist sie auch nachfrageseitig begründet durch die Alterung der Bevölkerung oder den medizinischen Fortschritt. Busse sieht die Ursache der Mengenausweitung mehrheitlich aufseiten des Angebots und weniger aufseiten der Nachfrage. So werden gemäss Busse ambulante Behandlungen viermal öfters durchgeführt, wenn die benötigte Infrastruktur in derselben Praxis bzw. Einrichtung vorzufinden ist (sog. «self-referral»), 
als wenn ein Patient zur externen Behandlung überwiesen werden muss. Deutsche Spitäler, die ihre Verweildauer besonders stark senkten, haben ihre Fallzahlen überdurchschnittlich gesteigert. Zudem stellte er fest, dass Veränderungen der DRG-Gewichte um 1\% kausal zu einer Veränderung der Fallzahlen um 0,2\% geführt haben. Abgesehen von diesen angebotsseitigen Ursachen verweist Busse jedoch auch auf nachfrageseitige Effekte wie die demographische Entwicklung und die Morbidität. Diese Faktoren erklärten in Deutschland jedoch nur rund ein Drittel der Fallzahlveränderungen zwischen 2007 und 2012, insbesondere weil die Morbidität bei Personen bis 75 Jahren abgenommen hat. Die Fachärzte Kaufmann und Heinz geben zu bedenken, dass die Erwartungen der Bevölkerung an die Medizin gestiegen sind. So falle ein Entscheid des Patienten für

\section{Medizinischer Fortschritt kostet nicht nur, sondern bringt Patienten gesteigerten Nutzen.}

eine Total-Endoprothese (TEP) oftmals schon in relativ jungen Jahren, um die privaten und beruflichen Aktivitäten nicht frühzeitig einschränken zu müssen, wie Heinz meint. Dass zu einem späteren Zeitpunkt mit einer unter Umständen wesentlich aufwendigeren Revisionsoperation zu rechnen ist, wird dabei in Kauf genommen. Kaufmann berichtet, dass Koronarangiographien heute viel öfters an Hochbetagten durchgeführt werden - auch weil es die Patienten und deren Angehörige wünschen. Damit wird der Einfluss der Alterung der Bevölkerung auf die Mengenentwicklung verstärkt - eine Entwicklung, die gemäss Busse auch durch die deutschen Zahlen untermauert wird.

\section{Ersichtliche Nutzensteigerung}

Neben den deutlich zunehmenden Fallzahlen und Kosten darf der höhere Nutzen nicht vergessen werden. Wie Kaufmann erläuterte, sank die Sterblichkeit beim akuten Herzinfarkt in Deutschland allen voran durch die flächendeckende Herzkatheter-Therapie um 40\% [2]. Gemäss den Qualitätsdaten des AQUA-Instituts [3] gäbe es demnach keine Überversorgung. Auch in der Schweiz hat die Sterblichkeit beim akuten Herzinfarkt deutlich abgenommen. Heinz ergänzt, dass die Schweiz laut einer Studie der OECD [4] im internationalen Vergleich eine tiefe Komplikationsrate ausweist.

Aus Sicht von Peters ist eine effektive Kosten-NutzenAnalyse äusserst wichtig. Er empfiehlt einen entsprechenden Ausbau der Health Technology Assessments (HTA) in der Schweiz.

\section{Lösungsvorschläge vorhanden - Begleitforschung zentral}

In der Diskussionsrunde besprachen die Referenten mit dem Plenum mögliche Lösungen. Busse brachte das Zweitmeinungsverfahren ins Spiel. Kaufmann und Heinz erachten dagegen Zweitmeinungen nur bei speziellen Fragestellungen und nicht generell als sinnvoll Sie geben zu bedenken, dass Patienten durch Zweitmeinungen teilweise verunsichert werden und demzufolge eine Drittmeinung einholen. Wichtig ist eine gute Kommunikation und Zusammenarbeit zwischen den Spezialistinnen sowie den Hausärzten: Letztere kennen die Patienten zumeist nicht nur am längsten, sondern auch am umfassendsten. Patienten sollten darüber informiert werden, dass die Krankenkassen die Zweitmeinungen bezahlen müssen, findet Peters. Er warnt jedoch ausdrücklich vor einem durchorganisierten Zweitmeinungsverfahren: Jeder Patient soll individuell vorgehen können.

Als weitere Lösungsvorschläge wurden die Angebotsplanung und eine bessere Koordination der Versorgung genannt. Für Busse und Peters wäre eine Angebotskonzentration wichtig, insbesondere in der hochspezialisierten Medizin. Aus Sicht von Kaufmann braucht es eine überregionale Planung, da kantonale Behörden durch lokalpolitische Faktoren und ihre Interessen als Spitalbetreiber in Konflikt geraten können.

Die Referenten und Tagungsteilnehmer finden es wichtig, dass auch in der Schweiz eine Begleitforschung zum Thema Mengenentwicklung unter DRG aufgebaut wird nur so können allfällige Fehlentwicklungen rechtzeitig erkannt werden. Laut Peters haben einige Parlamentarier entsprechende Vorstösse eingereicht; er hofft, dass der Bundesrat der Finanzierung der entsprechenden Studien zustimmen wird.

\section{Referenzen}

1 Schreyögg J, Busse R et al. Endbericht zum Forschungsauftrag zur Mengenentwicklung nach $\S 17 \mathrm{~b}$ Abs. 9 KHG. 2014. www.g-drg.de.

2 Pressetext DGK 01/2015 (2015), Aktueller Deutscher Herzbericht 2014: Deutsche Gesellschaft für Kardiologie.

3 Institut für angewandte Qualitätsförderung und Forschung im Gesundheitswesen.

4 OECD. Health at a Glance 2013: OECD Indicators, OECD Publishing.

Weitere Informationen zur FMH-Tagung

Alle Referate zur FMH-Tagung vom 12. Mai 2015 finden Sie über www.fmh.ch $\rightarrow$ Stationäre Tarife $\rightarrow$ Publikationen $\rightarrow$ FMH Tagung: Mengenausweitung aufgrund von DRG? 